\title{
Commercial Simplex and Multiplex PCR Assays for the Detection of Intestinal Parasites Giardia intestinalis, Entamoeba spp., and Cryptosporidium spp.: Comparative Evaluation of Seven Commercial PCR Kits with Routine In-House Simplex PCR Assays
}

\author{
Louise Basmaciyan 1,2,3,+(D), Alexandre François ${ }^{1,+}$, Anne Vincent ${ }^{1,2}$, Stéphane Valot ${ }^{1,2}$, Alain Bonnin ${ }^{1}$, \\ Damien Costa ${ }^{4,5,6} \mathbb{D}^{\text {, }}$, Romy Razakandrainibe ${ }^{4,6} \mathbb{D}$, Florent Morio ${ }^{7,8}$, Loic Favennec $4,5,6 \mathbb{D}$ and Frédéric Dalle ${ }^{1,2,3, *}$
}

1 Department of Parasitology /Mycology, University Hospital of Dijon, 21000 Dijon, France; louise.basmaciyan@chu-dijon.fr (L.B.); alexandre.francois@chu-dijon.fr (A.F.); anne.vincent@chu-dijon.fr (A.V.); stephane.valot@chu-dijon.fr (S.V.); alain.bonnin@chu-dijon.fr (A.B.)

2 CNR LE Cryptosporidiosis Collaborating Laboratory, Santé Publique France, 21000 Dijon, France

check for

updates

Citation: Basmaciyan, L.; François, A.; Vincent, A.; Valot, S.; Bonnin, A.; Costa, D.; Razakandrainibe, R.; Morio, F.; Favennec, L.; Dalle, F. Commercial Simplex and Multiplex PCR Assays for the Detection of Intestinal Parasites Giardia intestinalis, Entamoeba spp., and Cryptosporidium spp.: Comparative Evaluation of Seven Commercial PCR Kits with Routine In-House Simplex PCR Assays. Microorganisms 2021, 9, 2325. https://doi.org/10.3390/

microorganisms 9112325

Academic Editor: Nancy Guillén

Received: 15 October 2021

Accepted: 8 November 2021

Published: 10 November 2021

Publisher's Note: MDPI stays neutral with regard to jurisdictional claims in published maps and institutional affiliations.

Copyright: (C) 2021 by the authors Licensee MDPI, Basel, Switzerland. This article is an open access article distributed under the terms and conditions of the Creative Commons Attribution (CC BY) license (https:// creativecommons.org/licenses/by/ $4.0 /)$.
3 UMR PAM Univ Bourgogne Franche-Comté-AgroSup Dijon-Equipe Vin, Aliment, Microbiologie, Stress, 21078 Dijon, France

4 CNR LE Cryptosporidiosis, Santé Publique France, 76000 Rouen, France; damien.costa@chu-rouen.fr (D.C.); romy.razakandrainibe@univ-rouen.fr (R.R.); loic.favennec@chu-rouen.fr (L.F.)

5 Department of Parasitology/Mycology, University Hospital of Rouen, 76000 Rouen, France

6 EA ESCAPE 7510, University of Medicine Pharmacy Rouen, 76000 Rouen, France

7 Laboratorial de Parasitologie-Mycologie, Institut de Biologie, CHU de Nantes, 44000 Nantes, France; florent.morio@univ-nantes.fr

8 Département de Parasitologie et Mycologie Médicale, EA1155-IICiMed, Institut de Recherche en Santé 2, Université de Nantes, Nantes Atlantique Universités, 44035 Nantes, France

* Correspondence: frederic.dalle@chu-dijon.fr; Tel.: +33-(0)-380295014

$+\quad$ These authors are co-first authors on this work.

\begin{abstract}
Nowadays, many commercial kits allowing the detection of digestive parasites by DNA amplification methods have been developed, including simplex PCR assays (SimpPCRa) allowing the identification of a single parasite, and multiplex PCR assays (MultPCRa) allowing the identification of several parasites at once. Thus, aimed at improving the diagnosis of intestinal protozoal infections, it is essential to evaluate the performances of these new tools. A total of 174 DNA samples collected between 2007 and 2017 were retrospectively included in this study. Performances of four commercial SimpPCRa (i.e., CerTest-VIASURE ${ }^{\mathrm{TM}}$ ) and three MultPCRa (i.e., CerTest-VIASURE $^{\mathrm{TM}}$, FAST-TRACK-Diagnostics-FTD-Stool-Parasite ${ }^{\mathrm{TM}}$ and DIAGENODE-Gastroenteritis/Parasite-panel$\mathrm{I}^{\mathrm{TM}}$ ) were evaluated for the detection of Cryptosporidium spp., Entamoeba spp., and Giardia intestinalis in stool samples compared to our routinely used in-house SimpPCRa. Globally, the SimpPCRa showed better sensitivity/specificity for the detection of G. intestinalis, E. histolytica, E. dispar, and Cryptosporidium spp. (i.e., 96.9/93.6\%; 100/100\%; 95.5/100\%; and 100/99.3\%, respectively), compared to the three commercial MultPCRa tested. All in all, we showed that MultPCRa offer an interesting alternative for the detection of protozoans in stool samples depending on the clinical context.
\end{abstract}

Keywords: intestinal parasitic diseases; Entamoeba spp.; Giardia intestinalis; Cryptosporidium spp.; diagnosis; DNA amplification; stool samples; PCR

\section{Introduction}

Intestinal parasitic diseases (IPDs) are among the most important public health problems worldwide, affecting millions of people in developing countries. Moreover, IPDs are also observed in industrial countries, accounting for a significant morbidity and mortality 
worldwide [1-6]. Nowadays, due to (i) the movement of populations (e.g., travelers, international workers, and illegal migrants) and (ii) the wider use of immunosuppressing therapies, the number of cases of IPD diagnosed in European biological laboratories is increasing [7-10]. Parasitic diarrheas mainly involve intestinal protozoan parasites, including Giardia intestinalis, Cryptosporidium spp., and Entamoeba histolytica [4,11]. However, because of the lack of detection and surveillance of IPDs in developing countries, their clinical impact and their prevalence remain underestimated [4,12]. In this context, rapid and specific diagnosis methods for the detection of intestinal protozoan parasites is needed to (i) adapt treatment and (ii) adjust prevention strategies.

The reference method for the detection of intestinal protozoan parasites remains direct microscopic examination of stool samples, allowing the morphological identification of several protozoan parasites including G. intestinalis, Cryptosporidium spp., and Entamoeba spp. [13]. However, despite the use of staining and concentration methods aimed at improving their sensitivity, these conventional methods remain time-consuming with poor sensitivity that depends, among other things, on the operator's expertise [12]. Furthermore, microscopy does not allow the pathogenic E. histolytica to be distinguished from the non-pathogenic E. dispar parasite. Moreover, microscopic tools do not allow distinct species within the Cryptosporidium genus to be identified, which could have a real impact for epidemiological investigations (i.e., identification of the contamination source, outbreaks investigation, and number of cases [14-17]. Thus, to overcome the limitations of microscopy, alternative methods have been developed in the past few years including detection of parasitic antigens or DNA $[12,18]$.

Compared to conventional methods, DNA-based detection methods share numerous advantages for the detection of intestinal protozoan parasites in stool samples, including (i) a higher sensitivity and specificity, (ii) the ability to target multiple parasites (i.e., multiplex assays), and (iii) the ability to quantify and genotype parasitic DNA, as well as (iv) a faster turn-around time [18-20]. Many commercial kits allowing the detection of digestive parasites by DNA amplification methods have been developed. Among them, a wide range of commercial simplex PCR assays (SimpPCRa) have been developed for parasitic DNA detection, allowing the identification of a unique parasite. Recently, numerous multiplex PCR assays (MultPCRa), allowing the simultaneous detection of several parasites and for some of them, the quantification of parasitic DNA, have been commercialized [19]. Most of these MultPCRa target the intestinal protozoan parasites commonly involved in IPD (i.e., G. intestinalis, Cryptosporidium spp., and E. histolytica) [21-25]. However, those DNA-based methods display limitations which include (i) the difficulty of parasitic DNA extraction and (ii) the presence of PCR inhibitors in stool samples [21,22,26-29]. Moreover, the performances of the DNA-based methods performances vary depending on the amplification technology used (i.e., SybR Green, hybridization probe, or TaqMan ${ }^{\circledR}$ ). Thus, in order to improve the diagnosis of intestinal protozoan infections, it is essential to evaluate the performances of these new DNA amplification methods.

In this context, performances of four commercial SimpPCRa (i.e., simplex CerTestVIASURE $^{\mathrm{TM}}$ (San Mateo de Gállego Zaragoza, Spain) and three commercial MultPCRa (i.e., multiplex CerTest-VIASURETM (San Mateo de Gállego Zaragoza, Spain), Giardia/Entamoeba/ Cryptosporidium FAST-TRACK Diagnostics FTD Stool Parasite ${ }^{\text {TM }}$ (Esch-sur-Alzette, Luxembourg), and Giardia/Entamoeba/Cryptosporidium DIAGENODE-Gastroenteritis/Parasite-panel$\mathrm{I}^{\mathrm{TM}}$ (Liège, Belgium) were evaluated compared to our routinely used in-house SimpPCRa for the detection of Cryptosporidium spp., E. histolytica, E. dispar, and G. intestinalis.

\section{Materials and Methods}

\subsection{Sample Collection}

A total of 173 DNA samples, provided from the Parasitology Laboratories of Dijon University Hospital $(n=140)$, the National Reference Center-Expert Laboratory for Cryptosporidiosis (CNR-LE) (University Hospital of Rouen, France) $(n=31)$, and the Nantes University Hospital $(n=2)$, were retrospectively included in this study and stored 
at $-20{ }^{\circ} \mathrm{C}$ until PCR analysis. This DNA collection was obtained from stool samples formerly examined by microscopic methods for initial investigation. Overall, 86 samples were positive for at least one of the three protozoan parasites detected by the multiplex PCR assays evaluated in this study (i.e., G. intestinalis, Entamoeba spp., and Cryptosporidium spp.), 58 samples were negative for Giardia intestinalis, Entamoeba spp., and Cryptosporidium spp. but positive for other parasites, and 29 samples were negative for parasites (Table 1). The 58 samples negative for G. intestinalis, Entamoeba spp., and Cryptosporidium spp. but positive for other parasites were included in this study in order to evaluate eventual crossreactions. Thus, we selected a panel of nine helminths and six protozoa isolated in human stools (Table 2).

Table 1. DNA samples collected.

\begin{tabular}{cc}
\hline DNA Samples & $n$ \\
\hline Negative for G. intestinalis; Cryptosporidium spp.; E. histolytica; E. dispar but positive for other parasites & 29 \\
Positive for G. intestinalis * & 58 \\
Positive for E. histolytica * & 29 \\
Positive for E. dispar * & 5 \\
Positive for C. parvum * & 19 \\
Positive for C. hominis * & 10 \\
Positive for C. felis * & 10 \\
Positive for C. canis * & 4 \\
Positive for C. meleagridis * & 2 \\
Positive for C. ubiquitum * & 2 \\
Positive for G. intestinalis and Cryptosporidium sp. * & 2 \\
Positive for G. intestinalis and E. dispar & 1 \\
Total & 2 \\
& 173 \\
\hline
\end{tabular}

*: These samples were only positive for the given parasite.

Table 2. Gastrointestinal parasites included in the study to test for possible cross-reaction (i.e., not targeted by the different PCR assays evaluated in the study).

\begin{tabular}{|c|c|c|}
\hline & Genus/Species & $n$ \\
\hline \multirow{9}{*}{ 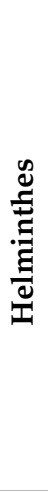 } & Hymenolepis nana & 9 \\
\hline & Shistosoma mansoni & 7 \\
\hline & Ankylostoma spp. & 8 \\
\hline & Enterobius vermicularis & 5 \\
\hline & Ascaris lumbricoides & 2 \\
\hline & Trichuris trichiura & 7 \\
\hline & Taenia spp. & 3 \\
\hline & Strongyloides stercoralis & 1 \\
\hline & Ankylostoma spp. + S. stercoralis & 1 \\
\hline \multirow{6}{*}{ 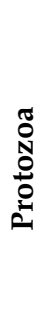 } & Cystoisospora belli & 3 \\
\hline & Blastocystis spp. & 2 \\
\hline & Chilomastix mesnilii & 1 \\
\hline & Entamoeba hartmani & 1 \\
\hline & Endolimax nana & 4 \\
\hline & Pentatrichomonas hominis & 1 \\
\hline
\end{tabular}


Table 2. Cont.

\begin{tabular}{|c|c|c|}
\hline & Genus/Species & $n$ \\
\hline \multirow{3}{*}{ 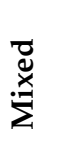 } & Endolimax nana + Enterobius vermicularis & 1 \\
\hline & Endolimax nana + Trichuris trichiura & 1 \\
\hline & Pentatrichomonas hominis + Trichuris trichiura & 1 \\
\hline & Total & 58 \\
\hline
\end{tabular}

\subsection{Stool DNA Extraction}

Stool DNA was extracted with the NucliSENS ${ }^{\circledR}$ easyMAG ${ }^{\circledR}$ automated system (BioMér ieux, Marcy-l'Etoile, France) following the protocol from Jeddi et al., 2013 [29]. Briefly, $400 \mathrm{mg}$ of stool sample was homogenized with $1 \mathrm{~mL}$ of NucliSENS ${ }^{\circledR}$ lysis buffer (BioMérieux, Marcy-l'Etoile, France) in a Lysing Matrix E tube (i.e., containing ceramic, silica and glass beads) (MP Biomedicals, Illkirch, France). It then underwent mechanical grinding using a FastPrep ${ }^{\circledR}-24$ (MP Biomedicals, Illkirch, France) at $6.0 \mathrm{~m} / \mathrm{s}$ for $1 \mathrm{~min}$. The stool suspension was then incubated at room temperature for $10 \mathrm{~min}$ before being centrifuged at $10,000 \times g$ for $10 \mathrm{~min}$. Finally, $250 \mu \mathrm{L}$ of supernatant was transferred in the DNA extraction NucliSENS $^{\circledR}$ easyMAG ${ }^{\circledR}$ automated system (BioMérieux, Marcy-l'Etoile, France) with $50 \mu \mathrm{L}$ of NucliSENS ${ }^{\circledR}$ EasyMAG $^{\circledR}$ magnetic silica (Biomérieux, Marcy-l'Etoile, France). Elution was performed at RT with $100 \mu \mathrm{L}$ of elution buffer. The eluted DNA volume obtained $(100 \mu \mathrm{L})$ was then stored at $-20{ }^{\circ} \mathrm{C}$.

\subsection{Commercial PCR Assays}

The VIASURE ${ }^{\mathrm{TM}}$ commercial SimpPCRa (San Mateo de Gállego Zaragoza, Espagne) (i.e., Giardia sp. CerTest VIASURE ${ }^{\mathrm{TM}}$, Cryptosporidium sp. CerTest VIASURE ${ }^{\mathrm{TM}}$, Entamoeba histolytica CerTest VIASURE ${ }^{\mathrm{TM}}$, and Entamoeba dispar CerTest VIASURE ${ }^{\mathrm{TM}}$ ), as well as the MultPCRa (i) Giardia/Entamoeba/Cryptosporidium CerTest VIASURE ${ }^{\mathrm{TM}}$ (San Mateo de Gállego Zaragoza, Espagne), (ii) Giardia/Entamoeba/Cryptosporidium DIAGENODE Gastroenteritis Parasite panel I ${ }^{\mathrm{TM}}$ (Liège, Belgium), and (iii) Giardia/Entamoeba/Cryptosporidium FAST-TRACK Diagnostics FTD Stool Parasite ${ }^{\mathrm{TM}}$ (Esch-sur-Alzette, Luxembourg), were performed on the LightCycler ${ }^{\circledR} 480$ automated system, (Roche Molecular Systems, Rotkreuz, Switzerland) according to the manufacturers' protocols.

The technical characteristics of all of the commercial PCR assays evaluated in the study are summarized in Supplementary Table S1.

\subsection{In-House Simplex PCR Assays for the Detection of Giardia intestinalis}

The in-house SimpPCRa for the detection of G. intestinalis was performed using SybrGreen method following the protocol from Verweij et al., 2003 [30]. Briefly, the amplification of a $116 \mathrm{bp}$ DNA fragment of the $18 \mathrm{~S}$ ribosomal rRNA gene was conducted using the following forward primers: GIA F1: $5^{\prime}$-gAC gCT CTC CCC AAg gA- $3^{\prime}$ and reverse primer GIA 127R: 5'-gTT gCC AgC ggT gTC C-3' and using the QuantiTect SYBR ${ }^{\circledR}$ Green PCR Kit polymerase (QIAGEN GmbH, Hilden, Germany). Amplification were performed on the LightCycler 2.0 Roche Molecular Systems, Inc. (Rotkreuz, Switzerland) in a final volume of $20 \mu \mathrm{L}$ containing $5 \mu \mathrm{L}$ of extracted DNA, $12 \mu \mathrm{L}$ of UTP-containing master mix, and $4 \mu \mathrm{L}$ of DNase/RNase free water. After a pre-incubation step at $95^{\circ} \mathrm{C}$ for $15 \mathrm{~min}$, the amplification was performed: denaturation at $95^{\circ} \mathrm{C}$ for $10 \mathrm{~s}$, and annealing/extension at $55^{\circ} \mathrm{C} / 72^{\circ} \mathrm{C}$, respectively, for 20 and $15 \mathrm{~s}$. Two negative (sterile water) and one positive controls were included in each assay. Samples were considered positive for targeted pathogens if $\mathrm{Ct}$ was equal to or below 40 cycles.

\subsection{In-House Simplex PCR Assays for the Detection of E. histolytica/dispar}

The in-house SimpPCRa for the detection and identification of E. histolytica/dispar was performed using a hybridization probe, and was adapted from Kebede et al., 2003 [31]. 
Briefly, a 120 bp DNA fragment of the $18 \mathrm{~S}$ rRNA gene was amplified using forward primers Ehd 74F 5'-AGTAGGATGAAACTGCGG-3' and reverse primer Ehd 259R 5'TTGTCGTGGCATCCTAA-3' . Detection used fluorescent-labelled probes Ehd sens $5^{\prime}$-fluoGGCCATTTTGTACTACAAACTATAGG- $3^{\prime}$ and Ehd anch $5^{\prime}$-Red $640^{\circledR}{ }^{\circledR}$-CGTCTCAAGTATT ATCTTTATCATTCACAAAGCTATCCT-ph- $3^{\prime}$. Ehd anch hybridizes in a conserved region among all Entamoeba species and Ehd-sens in a polymorphic region with mismatch between E.h. and E.d. The LightCycler ${ }^{\circledR}$ FastStart DNA Master HybProbe polymerase (ROCHE Diagnostics, GmbH, Mannheim, Germany) was used for this in-house PCR assay. Thermocycling and fluorescence detection were performed on the LightCycler 2.0 Roche Molecular Systems, Inc. (Rotkreuz, Switzerland) in a final volume of $20 \mu \mathrm{L}$ containing $5 \mu \mathrm{L}$ of extracted DNA samples and $15 \mu \mathrm{L}$ of UTP-containing master mix. After pre-incubation step at $95{ }^{\circ} \mathrm{C}$ for $10 \mathrm{~min}$, the amplification was performed: denaturation at $95{ }^{\circ} \mathrm{C}$ for $10 \mathrm{~s}$, touchdown annealing $\left(60\right.$ down to $\left.50{ }^{\circ} \mathrm{C}\right)$ for $15 \mathrm{~s}$ and extension at $72{ }^{\circ} \mathrm{C}$ for $15 \mathrm{~s}$. Two negative (sterile water) and two positive (E. histolytica and E. dispar) controls were included in each assay. The identification of the species E. histolytica or E. dispar in the case of a positive sample was made possible by the analysis of the melting curves (i.e., E. histolytica melting temperature: $62^{\circ} \mathrm{C}$; E. dispar melting temperature: $52^{\circ} \mathrm{C}$ ). Samples were considered positive for targeted pathogens if $\mathrm{Ct}$ was equal or below 40 cycles.

\subsection{In-House Simplex PCR Assays for the Detection of Cryptosporidium spp.}

The in-house SimpPCRa for the detection of Cryptosporidium spp. was performed using the hybridization probe format, following our protocol described in Brunet et al., 2016 [32]. Briefly, the amplification of a 258 bp DNA fragment of the 18S rRNA gene (GenBank accession $n^{\circ}$ L16996; positions 80 to 337) was conducted using the following primers and probes: 5'-GTTAAACTGCRAATGGCT-3'; $5^{\prime}$-CGTCATTGCCACGGTA-3' ${ }^{\prime} 5^{\prime}$-Red $640^{\circledR}$ gTCACATTAATTgTgATCCgTAAAg-ph; and 5'-CCgTCTAAAgCTgATAggTCAgAA ACTT gAATg-fluo. The LightCycler ${ }^{\circledR}$ FastStart DNA Master HybProbe polymerase (ROCHE Diagnostics GmbH, Mannheim, Germany) was used for this in-house PCR assay. Thermocycling and fluorescence detection were performed on the LightCycler 2.0 Roche Molecular Systems, Inc. (Rotkreuz, Switzerland) in a final volume of $20 \mu \mathrm{L}$ among which was $5 \mu \mathrm{L}$ of extracted DNA samples. After a pre-incubation step at $95^{\circ} \mathrm{C}$ for $10 \mathrm{~min}$, the amplification was performed: denaturation at $95^{\circ} \mathrm{C}$ for $10 \mathrm{~s}$, touchdown annealing $\left(60\right.$ down to $\left.50{ }^{\circ} \mathrm{C}\right)$ and extension at $72{ }^{\circ} \mathrm{C}$ for $15 \mathrm{~s}$. Two negative (sterile water) and two positive (C. parvum and C. hominis) controls were included in each assay. Samples were considered positive for targeted pathogens if $\mathrm{Ct}$ was equal or below 40 cycles. In the case of a positive sample, species identification was made possible by analysis of the melting curves (i.e., C. parvum melting temperature: $53.5^{\circ} \mathrm{C} ; \mathrm{C}$. hominis melting temperature: $61.5^{\circ} \mathrm{C} ; \mathrm{C}$. felis melting temperature: $48.5^{\circ} \mathrm{C} ; \mathrm{C}$. canis melting temperature: $51^{\circ} \mathrm{C} ; \mathrm{C}$. meleagridis melting temperature: $57^{\circ} \mathrm{C}$; and C. ubiquitum melting temperature: $53.5^{\circ} \mathrm{C}$ ).

\subsection{Design}

All the DNA samples included in the study were extracted from stools after formal microscopical examination and stored at $-20^{\circ} \mathrm{C}$ until their extraction. The volume of each DNA sample was sufficient to carry out all the 10 PCR assays along one single defrost cycle of up to $48 \mathrm{~h}$, avoiding a "DNA degradation" bias (i.e., freezing and thawing cycles having a detrimental effect on DNA preservation). Indeed, each sample underwent a single defrost cycle of up to $48 \mathrm{~h}$ during which time all molecular biology techniques were performed. The thermocycler LightCycler 2.0 (Roche Molecular Systems, Inc., Rotkreuz, Switzerland) was used for the in-house SimpPCRa. In accordance with the various supplier recommendations, the commercial kits were all evaluated using the same thermal cycler (LightCycler ${ }^{\circledR}$ 480 System, Roche Molecular Systems, Inc., Pleasanton, CA, USA). Then, data analysis was first performed using microscopical examination as the gold standard. All results were concordant between the in-house SimpPCRa and the microscopical examination (i.e., $n=173 / 173 ; 100 \%$ sensitivity $/ 100 \%$ specificity) and no PCR inhibitors having been 
detected using the commercial kit DIAControlDNA ${ }^{\mathrm{TM}}$ (Diagenode) as control of inhibition, the in-house SimpPCRa was considered to be as efficient as the microscopical examination, allowing us to use one or the other as gold standard. Thus, in order to compare each PCR assay with each other, the use of our in-house SimpPCRa as gold standard for data analysis was favored for this study (Figure 1).

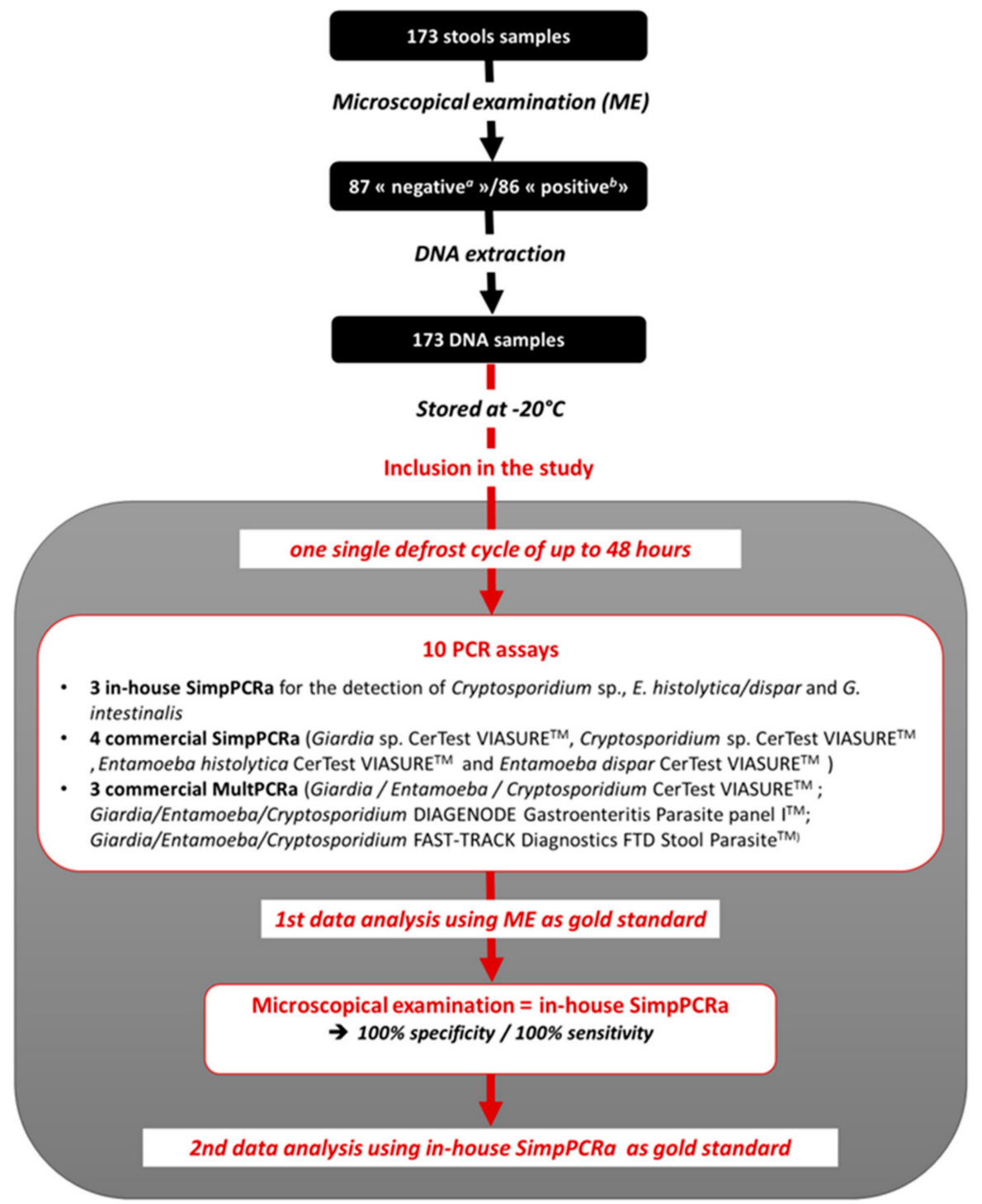

Figure 1. Flow chart.

\subsection{Statistical Analysis}

The statistical analysis was performed using the GraphPad PRISM software. The results of the commercial PCR assays were compared to the gold-standard (i.e., in-house PCR assay) using the Cohen's Kappa test. Cohen's Kappa ranges between 0 (no agreement between the two raters) and 1 (perfect agreement between the two raters). A Cohen's kappa value between 0.81 and 0.99 was considered as "near perfect agreement" while a Cohen's kappa value between 0.61 and 0.80 was considered as 'substantial agreement".

\section{Results}

The results of the seven commercial PCR assays for the detection of G. intestinalis, Entamoeba spp., and Cryptosporidium spp. were compared with those obtained with the Dijon University Hospital in-house simplex PCR assays. In order to compare the performances of each commercial PCR assay evaluated in this study, the sensitivity (Se), the specificity (Sp), 
the positive predictive value (PPV), and the negative predictive value (NPV) were calculated after sample classifications as: true positive samples (TP) (i.e., positive samples with both the in-house SimpPCRa and the commercial PCR assays were classified); false positive samples (FP) (i.e., positive samples by commercial PCR assays with a $\mathrm{Ct}<40$ cycles and negative by the in-house SimpPCRa); true negative samples (TN) (i.e., negative samples with both in-house SimpPCRa and the commercial PCR assays), and false negative samples (FN) (i.e., samples who were positive by in-house SimpPCRa but negative by commercial PCR assays). All the results are summarized in Tables 3 and 4.

Table 3. Results of the commercial simplex and multiplex PCR assays compared to Dijon University Hospital in-house simplex PCR assays.

\begin{tabular}{|c|c|c|c|c|c|c|}
\hline Kit & Parasites & $(+/+)$ & $(+/-)$ & $(-/+)$ & $(-I-)$ & Kappa Test \\
\hline \multirow{4}{*}{ CerTest VIASURE $^{\mathrm{TM}} \operatorname{SimpPCRa}$} & G. intestinalis & 31 & 1 & 9 & 132 & 0.8409 \\
\hline & E. histolytica & 5 & 0 & 0 & 168 & 1 \\
\hline & E. dispar & 20 & 1 & 0 & 152 & 0.9723 \\
\hline & Cryptosporidium spp. & 31 & 0 & 1 & 141 & 0.9806 \\
\hline \multirow{3}{*}{ CerTest VIASURE ${ }^{\mathrm{TM}}$ MultPCRa } & G. intestinalis & 26 & 6 & 2 & 139 & 0.8388 \\
\hline & E. histolytica & 5 & 0 & 0 & 168 & 1 \\
\hline & Cryptosporidium spp. & 31 & 0 & 1 & 141 & 0.9806 \\
\hline \multirow{3}{*}{$\begin{array}{l}\text { FAST-TRACK FTD Stool } \\
\text { parasites }{ }^{\mathrm{TM}} \mathrm{MultPCR} a\end{array}$} & G. intestinalis & 28 & 3 & 10 & 132 & 0.7653 \\
\hline & E. histolytica & 5 & 0 & 0 & 168 & 1 \\
\hline & Cryptosporidium spp. & 20 & 11 & 0 & 142 & 0.7490 \\
\hline \multirow{3}{*}{$\begin{array}{l}\text { DIAGENODE Gastroenteritis/Parasite } \\
\text { Panel I }{ }^{\mathrm{TM}} M u l t P C R a\end{array}$} & G. intestinalis & 26 & 8 & 4 & 135 & 0.7702 \\
\hline & E. histolytica & 5 & 0 & 0 & 168 & 1 \\
\hline & Cryptosporidium spp. & 23 & 8 & 1 & 141 & 0.8060 \\
\hline
\end{tabular}

$(+/+)$ : Positive by both in-house and commercial PCR assays (i.e., true positive sample, TP). (+/-): Positive by in-house PCR assays/negative by commercial PCR assays (i.e., false negative sample, FN). $(-/+)$ : Negative by in-house PCR assays/positive by commercial PCR assays (i.e., false positive sample, FP). $(-/-)$ : Negative by both in-house assays and commercial PCR assays (i.e., true negative sample, TN).

Table 4. Performances of the commercial simplex and multiplex PCR assays taking the Dijon University Hospital in-house simplex PCR assays as gold standard ( $n=173$ samples).

\begin{tabular}{|c|c|c|c|c|c|c|}
\hline Parasites & PCR Assay & Commercial Kit & Se & $\mathrm{Sp}$ & PPV & NPV \\
\hline \multirow{4}{*}{ Giardia intestinalis } & SimpPCRa & CerTest VIASURE $^{\mathrm{TM}}$ & 96.9 & 93.6 & 77.5 & 99.2 \\
\hline & \multirow{3}{*}{ MultPCRa } & CerTest VIASURE $^{\mathrm{TM}}$ & 81.2 & 98.6 & 92.9 & 95.9 \\
\hline & & FAST-TRACK FTD Stool parasites ${ }^{\mathrm{TM}}$ & 90.3 & 92.9 & 73.7 & 97.8 \\
\hline & & $\begin{array}{l}\text { DIAGENODE Gastroenteritis } \\
\text { Parasite Panel I TM }\end{array}$ & 76.5 & 97.1 & 86.7 & 94.4 \\
\hline \multirow{4}{*}{ Cryptosporidium sp. } & SimpPCRa & CerTest VIASURE $^{\mathrm{TM}}$ SimpPCRa & 100.0 & 99.3 & 96.9 & 100.0 \\
\hline & \multirow{3}{*}{ MultPCRa } & CerTest VIASURE ${ }^{\mathrm{TM}}$ & 100.0 & 99.3 & 96.9 & 100.0 \\
\hline & & FAST-TRACK FTD Stool parasites TM & 64.5 & 100.0 & 100.0 & 92.8 \\
\hline & & $\begin{array}{l}\text { DIAGENODE Gastroenteritis } \\
\text { Parasite Panel I TM }\end{array}$ & 74.2 & 99.3 & 95.8 & 94.6 \\
\hline \multirow{4}{*}{ Entamoeba histolytica } & SimpPCRa & CerTest VIASURE $^{\mathrm{TM}}$ & 100.0 & 100.0 & 100.0 & 100.0 \\
\hline & \multirow{3}{*}{ MultPCRa } & CerTest VIASURE $^{\mathrm{TM}}$ & 100.0 & 100.0 & 100.0 & 100.0 \\
\hline & & FAST-TRACK FTD Stool parasites TM & 100.0 & 100.0 & 100.0 & 100.0 \\
\hline & & $\begin{array}{l}\text { DIAGENODE Gastroenteritis } \\
\text { Parasite Panel I TM }\end{array}$ & 100.0 & 100.0 & 100.0 & 100.0 \\
\hline Entamoeba dispar & SimpPCRa & CerTest VIASURE $^{\mathrm{TM}}$ SimpPCRa & 95.5 & 100.0 & 100.0 & 99.3 \\
\hline
\end{tabular}

Se, sensitivity; Sp, specificity; NPV, negative predictive value; PPV, positive predictive value.

(i) Performances of commercial PCR assays for the detection of Giardia intestinalis.

The detection of G. intestinalis by the CerTest VIASURE ${ }^{\mathrm{TM}}$ SimpPCRa, the CerTest VIASURE $^{\mathrm{TM}}$ MultPCRa, the DIAGENODE Gastroenteritis Parasite panel IM ${ }^{\mathrm{TM}}$ MultPCRa, 
and the FAST-TRACK Diagnostics FTD Stool Parasite ${ }^{\mathrm{TM}}$ MultPCRa yielded a sensitivity/specificity of $96.9 / 93.6 \%, 81.2 / 98.6 \%, 90.3 / 92.9 \%, 76.5 / 97.1 \%$ and a NPV/PPV of $77.5 / 99.2 \%, 92.9 / 95.9 \%$, 73.7/97.8\%, and 86.7/94.4\%, respectively. All in all, nine samples were falsely negative (FN) for G. intestinalis detection with at least one of the commercial PCR assays tested in this study (Figure 2). Among them, three FN, which showed Ct equal or greater than 39 cycles with the in-house PCR (i.e., samples FN3, FN6, and FN9), were negative with all of the three MultPCRa, 2/3 samples being also negative with the CerTest VIASURE ${ }^{\mathrm{TM}}$ SimpPCRa. For the other six FN results, the samples displayed $\mathrm{Ct}$ values less than 36 cycles with in-house PCR assay (Table 5).

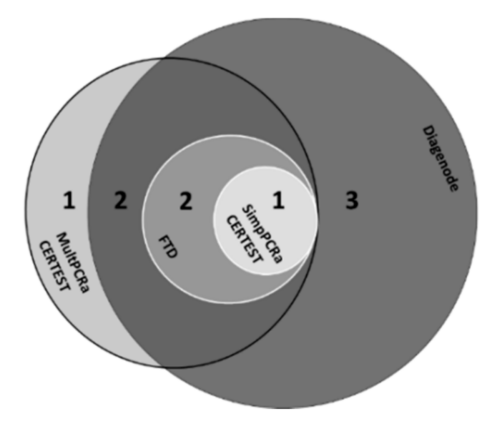

Figure 2. False-negative results for the detection of G. intestinalis using in-house SimPCRa as gold standard (Venn diagram) $(n=9)$.

Table 5. Cycle thresholds of the nine false-negative samples for G. intestinalis using in-house SimPCRa as gold standard.

\begin{tabular}{|c|c|c|c|c|c|c|c|c|c|}
\hline Samples & FN1 & FN2 & FN3 & FN4 & FN5 & FN6 & FN7 & FN8 & FN9 \\
\hline In-house SimpPCRa & 34.39 & 35.46 & 39.81 & 35.85 & 35.78 & 40 & 29.55 & 29.75 & 39 \\
\hline CerTest VIASURE SimpPCRa & 32.55 & 34.86 & neg & 35.82 & 33.05 & 36.97 & 31.66 & 29.11 & neg \\
\hline CerTest VIASURE MultPCRa & neg & neg & neg & neg & 35.09 & neg & 28.04 & 29.72 & neg \\
\hline FAST-TRACK FTD Stool parasites MultPCRa & 30.34 & 27.05 & neg & 28.54 & 27.71 & neg & 27.53 & 24.34 & neg \\
\hline DIAGENODE Gastroenteritis Parasite Panel I MultPCRa & 31.09 & neg & neg & neg & neg & neg & neg & neg & neg \\
\hline
\end{tabular}

Regarding the false positive samples, twelve samples were positive with at least one of the commercial PCR assays while they were negative with the in-house SimPCRa, with Ct varying from 27.2 to 37.77 (Table 6). Among those unexpected positive samples, one sample was positive with all the four commercial PCR assays (i.e., sample FP6), three samples were positive with three commercial PCR assays (i.e., samples FP2, FP5, and FP8), four samples were positive with two commercial PCR assays (i.e., samples FP7, FP9, FP10, and FP11), and four samples were positive with only one commercial PCR assay (i.e., samples FP1, FP3, FP4, and FP12). The best positivity rates were obtained with the FAST-TRACK Diagnostics FTD Stool Parasite ${ }^{\mathrm{TM}}$ MultPCRa followed by the CerTest VIASURE ${ }^{\mathrm{TM}}$ SimpPCRa detecting 10 and 9 of these unexpected positive samples, respectively.

Table 6. Cycle thresholds of the twelve positive samples for G. intestinalis with at least one of the commercial PCR assays but negative with the in-house SimPCRa (i.e., false positive samples).

\begin{tabular}{|c|c|c|c|c|c|c|c|c|c|c|c|c|}
\hline Samples & FP1 & FP2 & FP3 & FP4 & FP5 & FP6 & FP7 & FP8 & FP9 & FP10 & FP11 & FP12 \\
\hline In-house SimpPCRa & neg & neg & neg & neg & neg & neg & neg & neg & neg & neg & neg & neg \\
\hline CerTest VIASURE SimpPCRa & neg & 35.82 & neg & neg & 36.88 & 31.21 & 37.77 & 29 & 35.69 & 35.51 & 36.75 & 35.67 \\
\hline CerTest VIASURE MultPCRa & neg & neg & neg & neg & 35.28 & 27.2 & neg & neg & neg & neg & neg & neg \\
\hline $\begin{array}{l}\text { FAST-TRACK FTD Stool } \\
\text { parasites MultPCRa }\end{array}$ & 30.56 & 29.63 & 29.02 & 30.74 & neg & 29.28 & 30.54 & 27.81 & 30.82 & 30.68 & 30.07 & neg \\
\hline $\begin{array}{l}\text { DIAGENODE Gastroenteritis } \\
\text { Parasite Panel I MultPCRa }\end{array}$ & neg & 34.46 & neg & neg & 34.58 & 30.88 & neg & 29.8 & neg & neg & neg & neg \\
\hline
\end{tabular}


(ii) Performances of commercial PCR assays for the detection of Cryptosporidium spp.

The sensitivity/specificity for Cryptosporidium sp. detection by the CerTest VIASURE ${ }^{\mathrm{TM}}$ SimpPCRa, the CerTest VIASURE ${ }^{\mathrm{TM}}$ MultPCRa, the DIAGENODE Gastroenteritis Parasite panel $\mathrm{I}^{\mathrm{TM}}$ MultPCRa, and the FAST-TRACK Diagnostics FTD Stool Parasite ${ }^{\mathrm{TM}}$ MultPCRa were 100/99.3\%, 100/99.3\%,64.5/100\%, and 74.2/99.3\%, respectively, with PPV and NPV varying from $95.8 \%$ to $100 \%$ and $92.8 \%$ to $100 \%$, respectively. Among the 173 samples included in this study, all the commercial PCR assays tested in this study showed specificity greater than $99 \%$. Conversely, regarding the sensitivity, the performances of the commercial PCR assays tested varied from $64.5 \%$ to $100 \%$ according to the Cryptosporidium species (Figure 3). Indeed, while the CerTest VIASURE ${ }^{\mathrm{TM}}$ SimpPCRa and MultPCRa assays detected $100 \%(n=31)$ of the six Cryptosporidium species included in the study (i.e., C. hominis, C. paroum, C. canis, C. felis, C. meleagridis, and C. ubiquitum), the commercial DIAGENODE Gastroenteritis Parasite panel I ${ }^{\mathrm{TM}}$ showed poorer performances, detecting only three Cryptosporidium species (i.e., C. hominis, C. parvum, and C. meleagridis). Lastly, the FAST-TRACK Diagnostics FTD Stool Parasite ${ }^{\mathrm{TM}}$, although capable of detecting one more species than the DIAGENODE Gastroenteritis Parasite panel I ${ }^{\mathrm{TM}}$ (i.e., C. ubiquitum), displayed the worst performances by detecting only $80 \%(n=9 / 11)$ and $70 \%(n=7 / 10)$ of the two species most often isolated in human cryptosporidiosis, namely C. parvum and C. hominis, respectively, (Figure 3). For all the FN results, the samples had Ct less than 35 cycles with the in-house SimpPCRa.

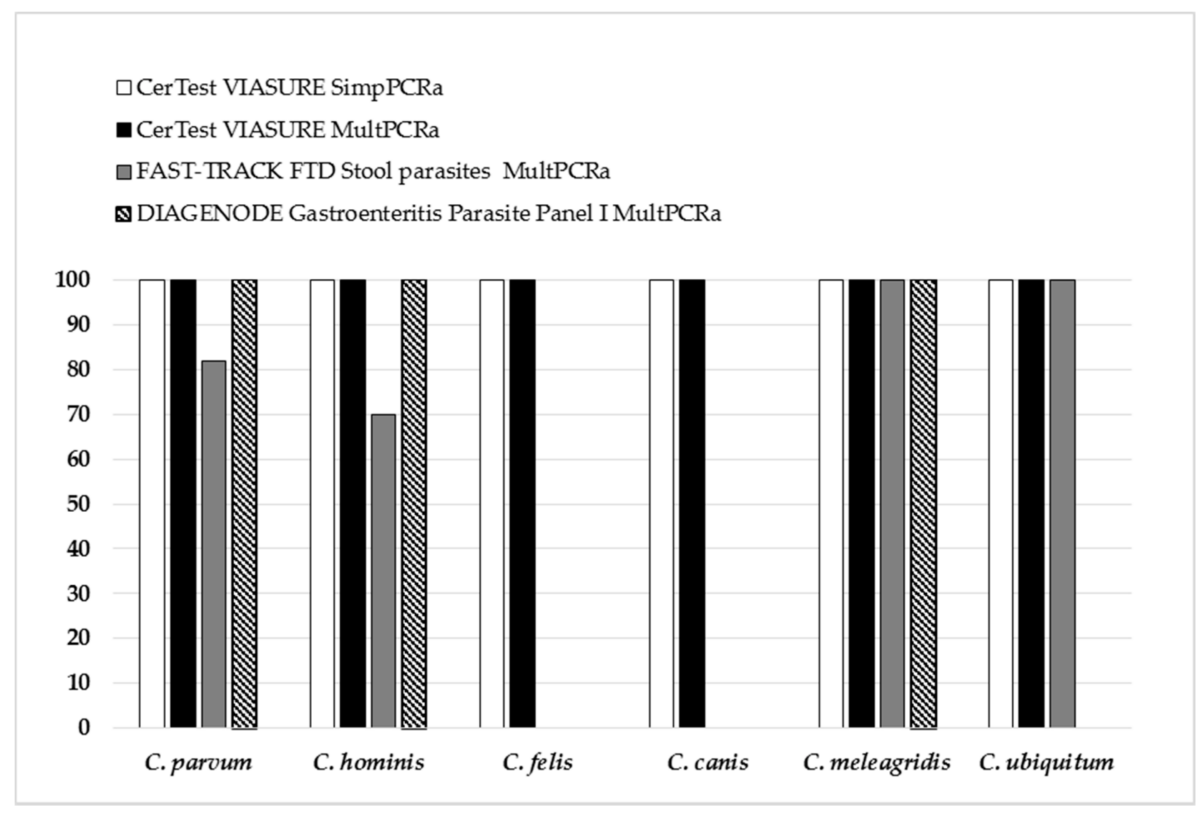

Figure 3. Performance (sensitivity) of the commercial PCR assays for the detection of Cryptosporidium spp. (C. parum, $n=10 ;$ C. hominis, $n=10 ;$ C. felis, $n=4 ;$ C. canis, $n=2 ;$ C. meleagridis, $n=2 ;$ C. ubiquitum, $n=2)$.

(iii) Performances of commercial PCR assays for the detection of Entamoeba spp.

Concerning E. dispar detection, only one commercial assay (i.e., the CerTest VIASURE ${ }^{\mathrm{TM}}$ SimpPCRa) was able to detect this species and the performance observed was globally good with 100/95.5\% specificity/sensitivity and 99.3/100\% NPV/PPV. Finally, the performances of all of the commercial PCR assays for the detection of Entamoeba histolytica were excellent, displaying 100/100\% specificity/sensitivity for all the commercial PCR assays tested in this study. 


\section{Discussion}

The aim of this study was to evaluate the performances of seven commercial PCR assays for the detection of the most common protozoa involved in human gastro-intestinal parasitic infections. The 173 DNA samples included in this study underwent a single freezing/thawing cycle, therefore avoiding the impact of storage on a possible DNA degradation of the samples. All in all, the seven commercial PCR assays showed very good specificity (i.e., $>99 \%$ ) associated with variable sensitivities depending on the parasite targeted (i.e., ranging from $64.5 \%$ to $100 \%$ ).

First for Entamoeba spp. detection, we observed a perfect match between all the commercial PCR assays tested for the detection of E. histolytica in stool samples with $100 / 100 \%$, sensibility/specificity. A limitation of our study is the small number of positive samples included (i.e., $n=5$ ), making the interpretation of this result difficult. However, the diagnosis of amoebiasis remaining difficult, the contribution of molecular biology is essential. Indeed, it has been shown that molecular methods had better performances for identification of E. histolytica compared to (i) antigen detection whose performances are poor $[21,33]$ or (ii) microscopic examination that does not allow the distinction between the causative agent of amoebiasis E. histolytica and the non-pathogenic E. dispar [17,34-37]. In this context, detection of E. histolytica DNA in stool samples would provide appropriate treatment to patients, even in the case of negative microscopic examination.

Second, regarding Cryptosporidium spp. detection, the performances of the PCR assays may depend on the Cryptosporidium species. In our sample collection, we selected six Cryptosporidium species isolated in humans: C. hominis, C. parvum, C. felis, C. meleagridis, C. canis, and C. ubiquitum [38]. The simplex and multiplex CerTest VIASURE ${ }^{\mathrm{TM}}$ commercial PCR assays were able to detect all of the six species of Cryptosporidium included in the study (i.e., $100 / 100 \%$ sensibility/specificity). By comparison, poor sensitivity of the multiplex DIAGENODE Gastroenteritis/Parasite panel $\mathrm{I}^{\mathrm{TM}}$ (i.e., $74.2 \%$ ) was attributable to the inability of this commercial PCR assay to detect C. felis, C. canis, and C. ubiquitum. However, the manufacturer's recommendations limit the use of the multiplex DIAGENODE Gastroenteritis/Parasite panel $\mathrm{I}^{\mathrm{TM}}$ commercial PCR assay to C. parvum detection. Thus, our results are in agreement with the manufacturer's recommendations, detecting $100 \%$ of C. parvum but also of $100 \%$ C. hominis and C. meleagridis. In addition, the multiplex FAST-TRACK Diagnostics FTD Stool Parasite ${ }^{\mathrm{TM}}$ commercial PCR assay showed the worst performances for the detection of Cryptosporidium spp., the two species most often isolated in human cryptosporidiosis, with C. hominis and C. parvum being detected in only $80 \%$ and $70 \%$ of cases, respectively, and none of the C. felis and C. canis having been detected. Surprisingly, no manufacturer's recommendations were available, specifying the species possibly detected by their assay. Thus, although our study only included a small number of positive samples per species, we highlighted discrepancies in the performances of the different multiplex commercial PCR assays for the detection of Cryptosporidium species, exposing a risk to diagnosis when using some PCR assays.

Finally, the most obvious performance discrepancies between the different commercial PCR assays were observed for the detection of G. intestinalis, with sensitivity and negative predictive value varying from $76.5 \%$ to $96.9 \%$ and $94.4 \%$ to $99.2 \%$, respectively. Focusing on the commercial multiplex PCR assays performances, our results are consistent with previous studies reporting sensitivities varying from $64 \%$ to $92 \%$ and from 92.7 to $100 \%$ for the DIAGENODE Gastroenteritis/Parasite panel IM ${ }^{\mathrm{TM}}$ commercial PCR assay and the FASTTRACK FTD Stool parasites ${ }^{\mathrm{TM}}$ commercial PCR assay, respectively, [21-25]. Regarding the twelve samples described in Table 6, they were classified as false positive based on the negative result obtained with the gold standard method. However, all those unexpected positive samples showed $\mathrm{Ct}$ lower than 37 cycles and valid amplification curves. Interestingly, focusing on the manufacturer's recommendations, the samples with a $\mathrm{Ct}<40$ cycles should be considered as positive samples. Moreover, more than half of these unexpected positive samples were positive with at least two PCR assays (i.e., $n=8 / 12$ ) suggesting that these unexpected positive samples could be finally true positive. 
All in all, we showed that the performances of the PCR assays are variable depending on the parasite target but also on the qPCR methods used. Indeed, among the amplification technologies tested, the SybR ${ }^{\circledR}$ Green and Hybridization Probe technologies allowed the generation of melting curves ensuring a good specificity (i.e., no false positives results). In parallel, although the Taqman ${ }^{\circledR}$ probe technology was less specific, it allowed multiplexing. Interestingly, it is well known that the PCR sensitivity depends on the target fragment copy number, however this information was unavailable for most of the commercial PCR assays tested, making it difficult to compare them to each other. Finally, no PCR inhibitor was detected when using the commercial kit DiaControlDNATM (Diagenode) as a control of inhibition. Knowing the lack of consensus on the method to use to detect the presence of PCR inhibitors, it would be interesting in the future to initiate work aimed at studying the different internal control formats, particularly in the complex matrix of stool samples. Finally, despite enhancement of the detection of parasites in stool samples, multiplex PCRs assays still remain complementary approaches to microscopic techniques since no multiplex PCR assay allow detection of all the parasites putatively involved in human pathology.

\section{Conclusions}

In conclusion, the commercial PCR assays showed satisfactory performances for the detection in stools samples of the three most common intestinal protozoa responsible for IPD in developed countries (i.e., Giardia intestinalis, Entamoeba histolytica, and Cryptosporidium spp.). Moreover, the multiplex PCR assays offer time-saving methods over microscopy while allowing molecular distinction of Entamoeba histolytica and Entamoeba dispar. Nowadays, microscopy-based techniques remain the gold standard for the detection of parasites in stools thanks to the exhaustivity of the pathogens targeted. However simplex and multiplex PCR assays offer interesting alternatives for the detection of digestive protozoans. Thus, positioning of multiplex PCR assays in the diagnostic strategy of IPD remains to be specified.

Supplementary Materials: The following are available online at https://www.mdpi.com/article/10 .3390 /microorganisms9112325/s1, Table S1: Technical characteristics of the commercial simplex and multiplex PCR assays evaluated in the study.

Author Contributions: Conceptualization, F.D. and L.B.; methodology, F.D., S.V. and L.B.; formal analysis, A.F. and L.B.; resources, L.F., F.D., D.C., R.R., S.V., L.B., A.V., A.B. and F.M.; writing-original draft preparation, L.B. and A.F.; writing-review and editing, L.B., S.V., A.B., D.C., F.M., L.F. and F.D.; visualization, A.F. and L.B.; supervision, F.D. and L.B. All authors have read and agreed to the published version of the manuscript.

Funding: This research was funded Certest Biotec SL.

Institutional Review Board Statement: Not applicable.

Informed Consent Statement: Not applicable.

Data Availability Statement: Not applicable.

Conflicts of Interest: The authors declare no conflict of interest. The funders had no role in the design of the study; in the collection, analyses, or interpretation of data; in the writing of the manuscript, or in the decision to publish the results.

\section{References}

1. Bercu, T.E.; Petri, W.A.; Behm, B.W. Amebic colitis: New insights into pathogenesis and treatment. Curr. Gastroenterol. Rep. 2007, 9, 429-433. [CrossRef]

2. Ali, I.K.M.; Clark, C.G.; Petri, W.A., Jr. Molecular epidemiology of amebiasis. Infect. Genet. 2008, 8, 698-707. [CrossRef]

3. Ximénez, C.; Morán, P.; Rojas, L.; Valadez, A.; Gómez, A. Reassessment of the epidemiology of amebiasis: State of the art. Infect. Evol. 2009, 9, 1023-1032. [CrossRef]

4. Fletcher, S.M.; Stark, D.; Harkness, J.; Ellis, J. Enteric protozoa in the developed world: A public health perspective. Clin. Microbiol. Rev. 2012, 25, 420-449. [CrossRef] 
5. Fletcher, S.M.; McLaws, M.-L.; Ellis, J.T. Prevalence of gastrointestinal pathogens in developed and developing countries: Systematic review and meta-analysis. J. Public Health Res. 2013, 2, 42. [CrossRef]

6. Abubakar, I.I.; Tillmann, T.; Banerjee, A. Global, regional, and national age-sex specific all-cause and cause-specific mortality for 240 causes of death, 1990-2013: A systematic analysis for the Global Burden of Disease Study 2013. Lancet 2015, 385, 117-171.

7. Gautret, P.; Cramer, J.P.; Field, V.; Caumes, E.; Jensenius, M.; Gkrania-Klotsas, E.; De Vries, P.J.; Grobusch, M.P.; Lopez-Velez, R.; Castelli, F.; et al. Infectious diseases among travellers and migrants in Europe, EuroTravNet 2010. Eurosurveillance 2012, $17,20205$. [CrossRef]

8. Troeger, H.; Epple, H.-J.; Schneider, T.; Wahnschaffe, U.; Ullrich, R.; Burchard, G.-D.; Jelinek, T.; Zeitz, M.; Fromm, M.; Schulzke, J.-D. Effect of chronic Giardia lamblia infection on epithelial transport and barrier function in human duodenum. Gut 2007, 56, 328-335. [CrossRef]

9. Shah, N.; DuPont, H.L.; Ramsey, D.J. Global etiology of travelers' diarrhea: Systematic review from 1973 to the present. Am. J. Trop. Med. Hyg. 2009, 80, 609-614. [CrossRef]

10. Thellier, M.; Bart-Delabesse, E.; Poupon, M.C.; Faussart, A. L'amoebose intestinale humaine revisitée: Entamoeba histolytica, pathogène, est moins fréquent que Entamoeba dispar, non pathogène. La Lett. L'infectiologue 2007, 22, 182-190.

11. Leitch, G.J.; He, Q. Cryptosporidiosis-an overview. J. Biomed. Res. 2011, 25, 1-16. [CrossRef]

12. McHardy, I.H.; Wu, M.; Shimizu-Cohen, R.; Couturier, M.R.; Humphries, R.M. Detection of intestinal protozoa in the clinical laboratory. J. Clin. Microbiol. 2014, 52, 712-720. [CrossRef]

13. Francis, J.; Barrett, S.P.; Chiodini, P.L. Best Practice No 174. Best practice guidelines for the examination of specimens for the diagnosis of parasitic infections in routine diagnostic laboratories. J. Clin. Pathol. 2003, 56, 888-891. [CrossRef]

14. Gonin, P.; Trudel, L. Detection and differentiation of Entamoeba histolytica and Entamoeba dispar isolates in clinical samples by PCR and enzyme-linked immunosorbent assay. J. Clin. Microbiol. 2003, 41, 237-241. [CrossRef]

15. Lebbad, M.; Svärd, S.G. PCR differentiation of Entamoeba histolytica and Entamoeba dispar from patients with amoeba infection initially diagnosed by microscopy. Scand. J. Infect. Dis. 2005, 37, 680-685. [CrossRef]

16. Hamzah, Z.; Petmitr, S.; Mungthin, M.; Leelayoova, S.; Chavalitshewinkoon-Petmitr, P. Differential detection of Entamoeba histolytica, Entamoeba dispar, and Entamoeba moshkovskii by a single-round PCR assay. J. Clin. Microbiol. 2006, 44, 3196-3200. [CrossRef]

17. Morio, F.; Valot, S.; Laude, A.; Desoubeaux, G.; Argy, N.; Nourrisson, C.; Pomares, C.; Machouart, M.; Le Govic, Y.; Dalle, F.; et al. Evaluation of a new multiplex PCR assay (ParaGENIE G-Amoeba Real-Time PCR kit) targeting Giardia intestinalis, Entamoeba histolytica and Entamoeba dispar/Entamoeba moshkovskii from stool specimens: Evidence for the limited performances of microscopy-based approach for amoeba species identification. Clin. Microbiol. Infect. 2018, 24, 1205-1209. [PubMed]

18. Verweij, J.J.; Stensvold, C.R. Molecular testing for clinical diagnosis and epidemiological investigations of intestinal parasitic infections. Clin. Microbiol. Rev. 2014, 27, 371-418. [CrossRef]

19. Binnicker, M.J. Multiplex molecular panels for the diagnosis of gastrointestinal infection: Performance, result interpretation and cost-effectiveness. J. Clin. Microbiol. 2015, 53, 3723-3728. [CrossRef]

20. Sow, D.; Parola, P.; Sylla, K.; Ndiaye, M.; Delaunay, P.; Halfon, P.; Camiade, S.; Dieng, T.; Tine, R.C.K.; Faye, B.; et al. Performance of real-time polymerase chain reaction assays for the detection of 20 gastrointestinal parasites in clinical samples from Senegal. Am. J. Trop. Med. Hyg. 2017, 97, 173-182. [CrossRef]

21. Laude, A.; Valot, S.; Desoubeaux, G.; Argy, N.; Nourrisson, C.; Pomares, C.; Machouart, M.; Le Govic, Y.; Dalle, F.; Botterel, F.; et al. Is real-time PCR-based diagnosis similar in performance to routine parasitological examination for the identification of Giardia intestinalis, Cryptosporidium parvum/Cryptosporidium hominis and Entamoeba histolytica from stool samples? Evaluation of a new commercial multiplex PCR assay and literature review. Clin. Microbiol. Infect. 2016, 22, e1-e8.

22. Van Lint, P.; Rossen, J.W.; Vermeiren, S.; Ver Elst, K.; Weekx, S.; Van Schaeren, J.; Jeurissen, A. Detection of Giardia lamblia, Cryptosporidium spp. and Entamoeba histolytica in clinical stool samples by using multiplex real-time PCR after automated DNA isolation. Acta Clin. Belg. 2013, 68, 188-192. [CrossRef]

23. Paulos, S.; Saugar, J.M.; de Lucio, A.; Fuentes, I.; Mateo, M.; Carmena, D. Comparative performance evaluation of four commercial multiplex real-time PCR assays for the detection of the diarrhoea-causing protozoa Cryptosporidium hominis/parvum, Giardia duodenalis and Entamoeba histolytica. PLoS ONE 2019, 14, e0215068. [CrossRef]

24. Autier, B.; Belaz, S.; Razakandrainibe, R.; Gangneux, J.-P.; Robert-Gangneux, F. Comparison of three commercial multiplex PCR assays for the diagnosis of intestinal protozoa. Parasite 2018, 25, 48. [CrossRef]

25. McAuliffe, G.N.; Anderson, T.P.; Stevens, M.; Adams, J.; Coleman, R.; Mahagamasekera, P.; Young, S.; Henderson, T.; Hofmann, M.; Jennings, L.C.; et al. Systematic application of multiplex PCR enhances the detection of bacteria, parasites, and viruses in stool samples. J. Infect. 2013, 67, 122-129. [CrossRef] [PubMed]

26. Liu, J.; Gratz, J.; Amour, C.; Kibiki, G.; Becker, S.; Janaki, L.; Verweij, J.J.; Taniuchi, M.; Sobuz, S.U.; Haque, R.; et al. A laboratorydeveloped TaqMan Array Card for simultaneous detection of 19 enteropathogens. J. Clin. Microbiol. 2013, 51, 472-480. [CrossRef] [PubMed]

27. Mejia, R.; Vicuña, Y.; Broncano, N.; Sandoval, C.; Vaca, M.; Chico, M.; Cooper, P.J.; Nutman, T.B. A novel, multi-parallel, real-time polymerase chain reaction approach for eight gastrointestinal parasites provides improved diagnostic capabilities to resource-limited at-risk populations. Am. J. Trop. Med. Hyg. 2013, 88, 1041-1047. [CrossRef] 
28. Stark, D.; Al-Qassab, S.E.; Barratt, J.L.N.; Stanley, K.; Roberts, T.; Marriott, D.; Harkness, J.; Ellis, J.T. Evaluation of multiplex tandem real-time PCR for detection of Cryptosporidium spp., Dientamoeba fragilis, Entamoeba histolytica, and Giardia intestinalis in clinical stool samples. J. Clin. Microbiol. 2011, 49, 257-262. [CrossRef] [PubMed]

29. Jeddi, F.; Piarrouxm, R.; Mary, C. Application of the NucliSENS easyMAG system for nucleic acid extraction: Optimization of DNA extraction for molecular diagnosis of parasitic and fungal diseases. Parasite 2013, 20, 52. [CrossRef]

30. Verweij, J.J.; Schinkelm, J.; Laeijendecker, D.; van Rooyenm, M.A.A.; van Lieshoutm, L.; Poldermanm, A.M. Real-time PCR for the detection of Giardia lamblia. Mol. Cell. Probes 2003, 17, 223-225. [CrossRef]

31. Kebede, A.; Verweij, J.; Dorigo-Zetsma, W.; Sanders, E.; Messele, T.; van Lieshout, L.; Petros, B.; Polderman, T. Overdiagnosis of amoebiasis in the absence of Entamoeba histolytica among patients presenting with diarrhoea in Wonji and Akaki, Ethiopia. Trans. R. Soc. Trop. Med. Hyg. 2003, 97, 305-307. [CrossRef]

32. Brunet, J.; Lemoine, J.P.; Pesson, B.; Valot, S.; Sautour, M.; Dalle, F.; Muller, C.; Borni-Duval, C.; Caillard, S.; Moulin, B.; et al. Ruling out nosocomial transmission of Cryptosporidium in a renal transplantation unit: Case report. BMC Infect. Dis. 2016, 16, 363. [CrossRef] [PubMed]

33. Korpe, P.S.; Stott, B.R.; Nazib, F.; Kabir, M.; Haque, R.; Herbein, J.F.; Petri, W.A. Evaluation of a rapid point-of-care fecal antigen detection test for Entamoeba histolytica. Am. J. Trop. Med. Hyg. 2012, 86, 980-981. [CrossRef] [PubMed]

34. Verweij, J.J.; Blangé, R.A.; Templeton, K.; Schinkel, J.; Brienen, E.A.T.; van Rooyen, M.A.A.; Van Lieshout, L.; Polderman, A.M. Simultaneous detection of Entamoeba histolytica, Giardia lamblia, and Cryptosporidium parvum in fecal samples by using multiplex real-time PCR. J. Clin. Microbiol. 2004, 42, 1220-1223. [CrossRef]

35. Stark, D.; van Hal, S.; Fotedar, R.; Butcher, A.; Marriott, D.; Ellis, J.; Harkness, J. Comparison of stool antigen detection kits to PCR for diagnosis of amebiasis. J. Clin. Microbiol. 2008, 46, 1678-1681. [CrossRef]

36. Solaymani-Mohammadi, S.; Rezaian, M.; Babaei, Z.; Rajabpour, A.; Meamar, A.R.; Pourbabai, A.A.; Petri, W.A., Jr. Comparison of a stool antigen detection kit and PCR for diagnosis of Entamoeba histolytica and Entamoeba dispar infections in asymptomatic cyst passers in Iran. J. Clin. Microbiol. 2006, 44, 2258-2261. [CrossRef]

37. Verkerke, H.P.; Hanbury, B.; Siddique, A.; Samie, A.; Haque, R.; Herbein, J.; Petri, W.A., Jr. Multisite clinical evaluation of a rapid test for Entamoeba histolytica in stool. J. Clin. Microbiol. 2015, 53, 493-497. [CrossRef]

38. Costa, D.; Razakandrainibe, R.; Valot, S.; Vannier, M.; Sautour, M.; Basmaciyan, L.; Gargala, G.; Viller, V.; Lemeteil, D.; Ballet, J.-J.; et al. Epidemiology of Cryptosporidiosis in France from 2017 to 2019. Microorganisms 2020, 8, 1358. [CrossRef] 